\title{
Experimental characterization of an ultrafast Thomson scattering $x$-ray source with three-dimensional time and frequency-domain analysis
}

\author{
W. J. Brown, S. G. Anderson, C. P. J. Barty, S. M. Betts, R. Booth, J. K. Crane, R. R. Cross, D. N. Fittinghoff, D. J. Gibson, \\ F. V. Hartemann, E. P. Hartouni, J. Kuba, G. P. Le Sage, D. R. Slaughter, A. M. Tremaine, A. J. Wootton, and P. T. Springer \\ Lawrence Livermore National Laboratory, Livermore, California 94550, USA \\ J. B. Rosenzweig \\ Department of Physics and Astronomy, University of California at Los Angeles, Los Angeles, California 90095, USA
}

(Received 30 January 2004; published 11 June 2004)

\begin{abstract}
We present a detailed comparison of the measured characteristics of Thomson backscattered $\mathrm{x}$ rays produced at the Picosecond Laser-Electron Interaction for the Dynamic Evaluation of Structures facility at Lawrence Livermore National Laboratory to predicted results from a newly developed, fully three-dimensional time and frequency-domain code. Based on the relativistic differential cross section, this code has the capability to calculate time and space dependent spectra of the $\mathrm{x}$-ray photons produced from linear Thomson scattering for both bandwidth-limited and chirped incident laser pulses. Spectral broadening of the scattered $\mathrm{x}$-ray pulse resulting from the incident laser bandwidth, perpendicular wave vector components in the laser focus, and the transverse and longitudinal phase spaces of the electron beam are included. Electron beam energy, energy spread, and transverse phase space measurements of the electron beam at the interaction point are presented, and the corresponding predicted $\mathrm{x}$-ray characteristics are determined. In addition, time-integrated measurements of the $\mathrm{x}$ rays produced from the interaction are presented and shown to agree well with the simulations.
\end{abstract}

DOI: 10.1103/PhysRevSTAB.7.060702

\section{INTRODUCTION}

The use of short laser pulses to generate high intensity, ultrashort x-ray pulses enables exciting new experimental capabilities, such as femtosecond pump-probe experiments used to temporally resolve structural dynamics of high- $Z$ materials on atomic (femtosecond) time scales $[1,2]$. The most promising methods for generating tunable, very high brightness electromagnetic radiation at short wavelengths $(<1 \AA)$ and pulse durations $(<1 \mathrm{ps})$ rely on either coherent radiation produced by $\mathrm{x}$-ray free-electron lasers, such as the planned Linac Coherent Light Source [3], or incoherent production through relativistic Thomson scattering, which has previously been employed for pioneering time-resolved diffraction measurements at LBNL [4,5], and is currently being investigated at several laboratories around the world [6-11]. Additionally, a growing number of research groups worldwide are exploring different $\mathrm{x}$-ray production mechanisms such as ultrafast, laser driven $K_{\alpha}$ sources [12] and electron bunch slicing in synchrotrons [13]. While coherent radiation sources generate higher power and narrower spectral bandwidths when compared to incoherent scattering, the potential of a Thomson source for high peak brightness within a relatively compact and affordable system makes it an attractive alternative for many applications.

The capability to accurately predict the spatial, spectral, and temporal characteristics of Thomson backscattered $\mathrm{x}$ rays is crucial for both the design of successful Thomson x-ray sources, as well as future experiments
PACS numbers: 41.60.-m, 52.59.-f, 41.75.-i, 52.38.-r

and applications utilizing such sources. While the theory of Thomson backscattered radiation is well known and has been documented extensively [14-20], there remains a need to have a complete three-dimensional (3D) timeresolved computational capability for the full determination of the temporally and spatially resolved spectra and intensity distributions produced from a Thomson interaction for arbitrary geometries. In particular, this capability will greatly benefit the understanding of the production of $\mathrm{x}$-ray pulses with chirped (or time correlated) spectra [21-24], potentially enabling significant improvement in the time resolution of femtosecond pump-probe experiments. With this motivation, a newly developed fully 3D time and frequency-domain code used for calculations of Thomson scattering of a short, intense laser pulse with a relativistic electron bunch has been developed at Lawrence Livermore National Laboratory. The details of this code, and the underlying theory, are presented in a companion paper [25].

In this paper, we present a comparison of timeintegrated spatial and spectral intensity measurements performed at the Picosecond Laser-Electron Interaction for the Dynamic Evaluation of Structures (PLEIADES) Thomson $\mathrm{x}$-ray source to $\mathrm{x}$-ray production simulations produced with the 3D time and frequency-domain code. This benchmarking proves important for both verifying and understanding the characteristics of the x-ray beam produced at the PLEIADES facility, as well as for verifying the validity of the theory used to develop the computer code. The $\mathrm{x}$ rays are produced by the collision of a $0.3 \mathrm{nC}, 50-60 \mathrm{MeV}$ electron bunch with a terawatt (TW) 
$800 \mathrm{~nm}$ laser pulse to produce peak $\mathrm{x}$-ray energies in the 60-70 keV range. In this paper, electron beam energy, energy spread, and transverse phase space measurements of the electron beam at the interaction point are presented, and the predicted $\mathrm{x}$-ray characteristics based on these measurements are determined. In addition, timeintegrated measurements of the $\mathrm{x}$ rays produced from the interaction are presented and shown to agree well with the simulations.

\section{3D TIME AND FREQUENCY-DOMAIN THEORY}

In this section, a brief overview of the theory behind the time and frequency-domain code is presented. For a single photon colliding with a plane wave, the number of scattered photons per unit solid angle, per unit frequency, per unit time is given by

$$
\begin{aligned}
\frac{d N_{1}}{d \omega_{s} d \Omega d t}(\theta, \phi)= & c\left(1-\boldsymbol{\beta}_{e} \cdot \mathbf{k}_{0} \frac{c}{\omega}\right) n_{\gamma} \frac{d \sigma}{d \Omega d \omega_{s}} \\
= & c\left(1-\boldsymbol{\beta}_{e} \cdot \mathbf{k}_{0} \frac{c}{\omega}\right) n_{\gamma}\left(\mathbf{r}_{e}, t\right) \frac{d \sigma}{d \Omega}(\theta, \phi) \\
& \times \delta\left[\omega_{s}-\omega_{0} g(\theta)\right],
\end{aligned}
$$

where $\sigma$ is the Thomson cross section, $n_{\gamma}$ is the photon density at the position $\mathbf{r}_{e}$ of the electron, $\omega_{0}$ is the incident electromagnetic wave (or photon) frequency, $\mathbf{k}_{0}$ is the incident wave vector, $\boldsymbol{\beta}_{e}$ is the velocity of the electron normalized to the speed of light $c, \omega_{s}$ is the scattered photon frequency, and $g(\theta)$ is the geometry dependent relativistic doppler up-shift of the scattered photon colliding with a plane wave, given by

$$
g(\theta)=\frac{\gamma\left(1-\frac{c}{\omega_{0}} \boldsymbol{\beta}_{e} \cdot \mathbf{k}_{0}\right)}{1-\beta \cos (\theta)} \approx 2 \gamma^{2} \frac{\left(1-\frac{c}{\omega_{0}} \boldsymbol{\beta}_{e} \cdot \mathbf{k}_{0}\right)}{1+\theta^{2} \gamma^{2}},
$$

where $\theta$ is the angle of the scattered photon direction from the direction of the electron in the lab frame. Under the paraxial approximation $[26,27]$, the spatial density of photons in the incoming laser beam can be represented by the expression

$$
n_{\gamma}(\mathbf{r}, t)=\frac{N_{\gamma} \exp \left[\frac{-2\left(c t+z_{L}\right)^{2}}{c^{2} \Delta t^{2}}\right]}{\left(\frac{\pi}{2}\right)^{3 / 2} c \Delta t w_{0}\left(1+z_{L}^{2} / z_{R}^{2}\right)} \exp \left[\frac{-2 r_{L}^{2}}{w_{0}\left(1+z_{L}^{2} / z_{R}^{2}\right)}\right],
$$

where $N_{\gamma}$ is the total number of photons in the bunch, $\mathrm{z}_{\mathrm{R}}=\pi w_{0}^{2} / \lambda_{0}$ is the Rayleigh range of the laser focus, and we have utilized a coordinate system $\left(x_{L}, y_{L}, z_{L}\right)$ such that the $z_{L}$ axis is antiparallel to the laser beam direction.

Equation (1) gives the complete spectral, spatial, and temporal properties of the scattered $\mathrm{x}$-ray beam for the case of a single electron colliding with a plane wave and represents the basis of the 3D time and frequency-domain code. To account for the laser pulse bandwidth, as well as the distribution of perpendicular components of the wave vector $k_{\perp}$ due to laser focusing, Eq. (1) is integrated over both the time-dependent laser bandwidth, represented by

$$
n_{\omega}[\omega, \Delta \omega, \zeta(t)]=\sqrt{\frac{2}{\pi}} \frac{1}{\Delta \omega_{0}(\zeta)} \exp \left\{-\frac{2\left[\omega-\omega_{0}(\zeta)\right]^{2}}{\Delta \omega_{0}^{2}(\zeta)}\right\},
$$

and the perpendicular wave vector components, which, assuming a radially symmetric focus, can be represented by the distribution function

$$
f_{k \perp}\left(k_{r}\right)=\frac{w_{0}^{2}}{2 \pi} \exp \left(-w_{0}^{2} k_{r}^{2}\right) .
$$

In Eq. (5), $w_{0}$ is the $1 / e^{2}$ intensity radius of the laser pulse, and perfect coherence has been assumed. In Eq. (4), $\zeta \equiv z_{L}+c t$ represents the longitudinal position within the laser pulse, while $\omega_{0}(\zeta)$ and $\Delta \omega_{0}(\zeta)$ represent the center frequency and $1 / e^{2}$ spectral bandwidth at a given $\zeta$. For the case of a bandwidth-limited pulse duration (i.e., no $\zeta$ dependence), the spectral bandwidth is given by the Fourier limit relation,

$$
\Delta \omega_{0} \Delta t_{0}=2,
$$

where $\Delta t_{0}$ is the $1 / e^{2}$ temporal width and a Gaussian distribution has been assumed. The total scattered photon spectral density flux for a single electron can then be obtained by performing the integration

$$
\frac{d N_{e}}{d \Omega d \omega_{s} d t}=\iint f_{k \perp} n_{\omega 0}\left(\mathbf{r}_{e}(t), t\right) \frac{d N_{1}}{d \omega_{s} d \Omega d t} d^{2} k_{\perp} d \omega_{0},
$$

where $N_{e}$ represents the total number of scattered photons for a single electron. The integration over $\omega_{0}$ is performed analytically to obtain

$$
\begin{aligned}
\frac{d N_{e}}{d \Omega d \omega_{s} d t}= & \sqrt{\frac{2}{\pi}} \int \frac{d N_{1}}{d \Omega d t} \frac{f_{k \perp}}{\Delta \omega_{0}(\zeta)\left|g\left(k_{\perp}, \theta\right)\right|} \\
& \times \exp \left\{-\frac{2\left[\omega_{s}-\omega_{0}(\zeta) g\left(k_{\perp}, \theta\right)\right]^{2}}{g^{2}\left(k_{\perp}, \theta\right) \Delta \omega_{0}^{2}(\zeta)}\right\} d^{2} k_{\perp},
\end{aligned}
$$

where $\left(d N_{1} / d \Omega d t\right)$ is the spectrally integrated photon density flux for the case of a single electron colliding with a plane wave and is equal to the right-hand side of Eq. (1) without the presence of the delta function. The presence of the laser bandwidth simply results in a corresponding width in the Doppler shifted $\mathrm{x}$-ray spectrum, while the effect of $k_{\perp}$ is to shift the maximum scattered photon energy due to the slight change in the relative incident angle between the electron and the incident wave vector, which leads to the spreading of the observed spectrum in a given direction.

Finally, to account for the effects of the electron beam energy spread and emittance (i.e., spread in the direction of different electrons), the electron beam can be represented by a series of macroparticles, and the results from each electron in a bunch are summed resulting in 


$$
\frac{d N_{T}\left(\theta, \phi, \omega_{s}, t\right)}{d \Omega d \omega_{s} d t}=\sum_{e} \frac{q_{e}}{e} \frac{d N_{e}\left[\theta_{e}(\theta, \phi), \phi_{e}(\theta, \phi), \omega_{s}, t\right]}{d \Omega d \omega_{s} d t},
$$

where $q_{e}$ is the charge represented by the macroparticle, and $\theta_{e}$ and $\phi_{e}$ represent the scattering angles of the photons in a rotated lab frame $\left(x_{e}, y_{e}, z_{e}\right)$ such that the $z_{e}$ axis is collinear with each electron and can be expressed in nonrotated coordinates through a simple rotation. The primary broadening mechanism is typically due to the electron beam divergence, which results in a shift in the center direction of the scattered $\mathrm{x}$-ray distribution for each electron, resulting in a corresponding spread in $\mathrm{x}$-ray energies at any given observation point. In addition, if the electron beam divergence is significant in comparison to the characteristic $1 / \gamma$ angular radiation width from a single electron, the overall divergence of the $\mathrm{x}$-ray beam will also be dependent on the details of the transverse electron beam phase space. Equation (9) is valid provided the scattered $\mathrm{x}$-ray beam can be considered to be incoherent, which is true provided the dimension of the electron bunch is much larger than the scattered x-ray wavelength.

The expression for $d \sigma / d \Omega$ in the lab frame is a relatively complicated expression for arbitrary interaction geometries and laser polarizations, but can be approximated in the high $\gamma$ limit to be [25]

$$
\frac{d \sigma}{d \Omega} \approx r_{0}^{2} \frac{4 \gamma^{2}}{\left(1+\theta^{2} \gamma^{2}\right)^{2}}\left[1-\frac{4\left(\theta^{2} \gamma^{2}\right)}{\left(1+\theta^{2} \gamma^{2}\right)^{2}} \sin ^{2}(\phi)\right],
$$

where $r_{0}$ is the classical electron radius, $\theta$ and $\phi$ are the scattering angles of the $\mathrm{x}$ rays with respect to the electron direction, and it is assumed that the laser is polarized in the plane where $\phi=\pi / 2$. A complete derivation of the general form of the cross section can be found in the companion paper [25], where a more complete discussion on the general properties of Thomson radiation can also be found.

Equation (9) represents the basic algorithm for the 3D time and frequency-domain code. It is both fully three dimensional, taking into account effects from the laser and electron beam $6 \mathrm{D}$ phase space at the interaction, and completely time and frequency resolved, allowing computation of the temporally and spatially dependent spectra for arbitrary interaction geometries. The background motion of the electron through the laser pulse is assumed to be ballistic, and the temporal information of the $x$-ray pulse is determined by calculating the time of flight of the scattered photon to a detector at a specified distance to the interaction at each time step in the simulation. Spatial information of the scattered x-ray pulses is determined by performing this calculation for several different observation directions specified by $\theta$ and $\phi$. The assumptions inherent in the 3D time and frequency-domain code include (i) the normalized vector potential of the incident laser pulse, $e A / m c$, is much less than 1 , (ii) the incident photon energy in the electron rest frame is much less than the electron rest mass (i.e., $\hbar \omega_{0}^{\prime} \ll m c^{2}$ ), and (iii) the scattered $x$-ray wavelength is much shorter than the size of the electron bunch (i.e., incoherent scattering).

\section{BENCHMARKING THE CODE}

To benchmark the code, $\mathrm{x}$-ray data taken from the PLEIADES Thomson $x$-ray source at LLNL [11] is compared to simulated results, given the measured electron beam and laser beam parameters. PLEIADES is a high brightness 10-100 keV Thomson $\mathrm{x}$-ray source designed for single-shot diffraction and radiography experiments in high- $Z$ materials. The facility includes an $S$-band $\mathrm{rf}$ photoinjector, a $100 \mathrm{MeV} S$-band accelerator, and a TWclass, $800 \mathrm{~nm}$ Ti:sapphire, chirped-pulse amplification (CPA) [28] laser system capable of delivering up to $500 \mathrm{~mJ}$ of energy in a $50 \mathrm{fs}$ Fourier transform-limited pulse. The $81.557 \mathrm{MHz}$ Kerr-lens mode-locked oscillator at the front end of the laser system serves as the master clock of the experiment. A single pulse from the oscillator is selected and amplified by the CPA and two four pass amplifiers to produce the drive pulse for the Thomson interaction. A second seed pulse is selected from the oscillator and transported to a separate CPA and frequency tripler to produce the drive pulse for the photocathode $\mathrm{rf}$ gun. The rf photoinjector used to produce the electron beam for PLEIADES is based on a 1.6-cell standing-wave geometry. The $266 \mathrm{~nm}$ UV laser profile at the gun cathode is a $2 \mathrm{~mm}$ diameter apertured Gaussian with a 3 ps rms temporal pulse length. The electron charge is typically about $250 \mathrm{pC}$, and the energy of the electron bunch out of the gun is about $3.5 \mathrm{MeV}$. The beam generated by the photoinjector is then accelerated to energies ranging between 20 and $100 \mathrm{MeV}$ by four $2.5 \mathrm{~m}$, SLAC-type traveling-wave accelerating sections. The experiment operates at a $10 \mathrm{~Hz}$ repetition rate.

Figure 1 shows a schematic of the interaction region. The electron beam is incident from the right and is focused by a quadrupole triplet with a focal length of about $25 \mathrm{~cm}$. The laser beam, incident from the left and polarized in the plane of the image, is focused with an off-axis parabola with a focal length of $1.5 \mathrm{~m}$. The focus is folded by a $0.5 \mathrm{in}$. BK7 flat placed directly in the beam line. The $F$ number of the focus is about 25 , and the measured $M^{2}$ of the laser is 1.6 , leading to a $1 / e^{2}$ intensity radius of $36 \mu \mathrm{m}$. The $\mathrm{x}$ rays produced in the interaction travel in the direction of the electron beam, while the electron beam itself is bent by a dipole magnet and transported to a shielded beam dump. The $\mathrm{x}$ rays pass through the laser turning optic (0.5 in. BK7 flat) and are detected by a CsI(Tl) scintillator fiber coupled to a 16 bit charge-coupled device (CCD) camera with a 3:1 taper. The CCD array has a pixel count of $1340 \times 1300$, with a pixel size of $20 \times 20 \mu \mathrm{m}$. This corresponds to an 


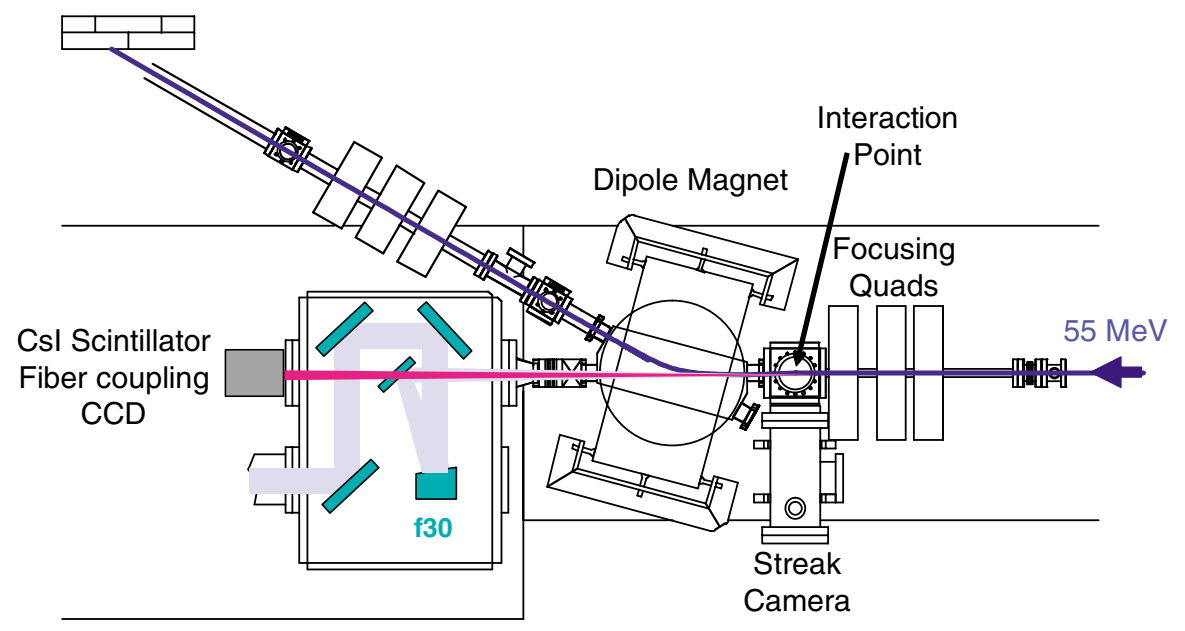

FIG. 1. (Color) Schematic of the interaction region for the PLEIADES Thomson x-ray source.

effective size of $60 \times 60 \mu \mathrm{m}$ at the scintillator, yielding a total detection area of about $63 \mathrm{~cm}^{2}$. With the CCD placed $1.82 \mathrm{~m}$ from the interaction, this corresponds to a detectable solid angle of about $43 \times 43 \mathrm{mrad}^{2}(0.019 \mathrm{sr})$. The CCD was calibrated with a $60 \mathrm{keV}{ }^{241} \mathrm{Am}$ source, yielding a sensitivity of 7.4 counts/photon, or correspondingly, 0.12 counts $/ \mathrm{keV}$ at $60 \mathrm{keV}$.

Figure 2(a) shows a typical x-ray profile detected with the $\mathrm{x}$-ray CCD. This image was produced by the collision of a $57 \mathrm{MeV}, 0.25 \mathrm{nC}$ electron bunch focused to an rms spot size of about $50 \mu \mathrm{m}$. The peak x-ray energy is about $77 \mathrm{keV}$. The laser pulse contained about $400 \mathrm{~mJ}$ of energy with a center wavelength near $810 \mathrm{~nm}$ and was focused to an rms spot size of about $18 \mu \mathrm{m}$, or $36 \mu \mathrm{m} 1 / e^{2}$ intensity radius. Figure 2(b) shows the intensity of the x-ray spot integrated along the $y$ (vertical) axis vs the divergence angle along the $x$ (horizontal) axis, where $\theta$ was calculated from the transverse position on the CCD scintillator and the distance of the CCD from the interaction point. It is seen that the intensity profile is Gaussian in shape. Because of the broadening of the $\mathrm{x}$-ray intensity profile induced by the divergence of the electron beam, as well as the attenuation through the laser turning optic of the low energy $x$ rays that comprise most of the energy in the wings of the angular profile, the measured intensity profile differs from the theoretical Lorentzian intensity profile for a single electron. An integration of the Gaussian profile results in an estimated total count number in the CCD image of $4.5 \times 10^{6}$. A very crude estimate of the total energy in the pulse can be reached by simply dividing this number by the single wavelength calibrated sensitivity $\eta$ (shown in Table I) yielding $3.7 \times 10^{7} \mathrm{keV}$. As discussed in Sec. III B, however, an accurate estimate will require full knowledge of the spectrum of the $\mathrm{x}$-ray pulse.

In this section, two comparisons between measurements of the x-ray beam characteristics and theoretical predictions from the time and frequency-domain code are

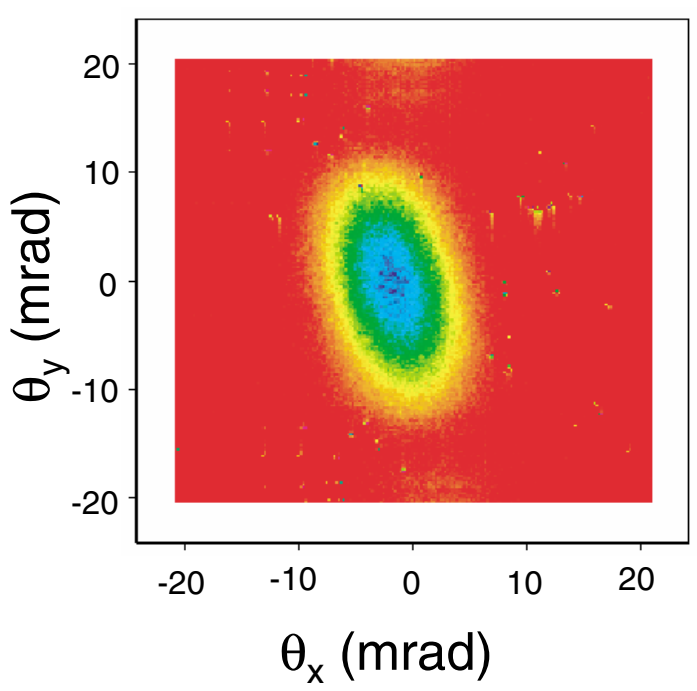

(a)

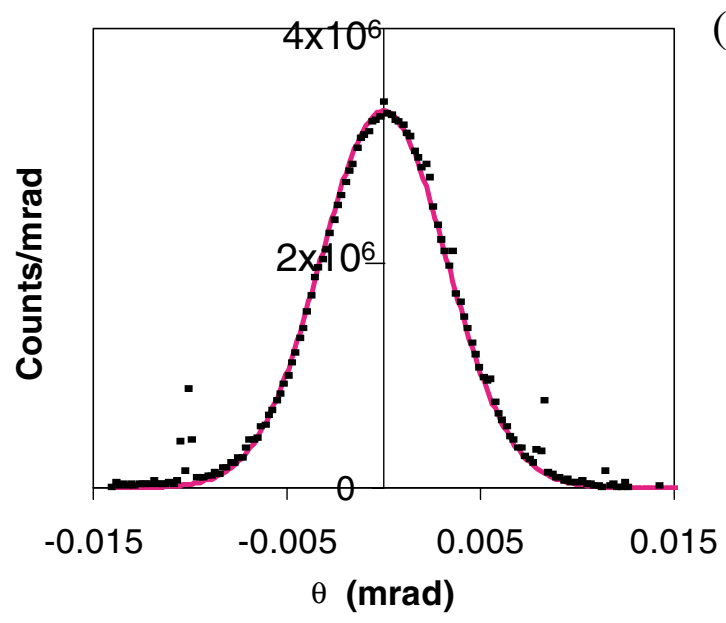

(b)

FIG. 2. (Color) Single shot $x$-ray beam image detected with the $\mathrm{x}$-ray CCD (a), and $y$ integrated background subtracted intensity profile vs the divergence angle in $x$ for the same image (b). 
TABLE I. X-ray CCD specifications.

\begin{tabular}{lc}
\hline \hline \multicolumn{1}{c}{ Parameter description } & Value \\
\hline Effective CCD array pixel size & $60 \mu \mathrm{m}$ \\
Calibrated sensitivity $\eta$ (at $60 \mathrm{keV})$ & $0.12 \mathrm{counts} / \mathrm{keV}$ \\
Dynamic range & $2^{16}$ \\
\hline \hline
\end{tabular}

presented. First, we compare the measured x-ray beam profile shown in Fig. 2 to the simulated profile, where the measured electron beam characteristics were used to determine the input parameters of the code. Next, the simulated and measured effect of a $K_{\alpha}$ filter on the intensity profile are compared as a means of testing the spatially correlated spectral content predicted by the calculations. It is shown that in both cases, good agreement between measurement and theory is observed.

\section{A. Electron beam diagnostics and reconstruction}

The sensitivity of the scattered $\mathrm{x}$-ray spectrum to the divergence of the electron beam necessitates an accurate understanding of the electron beam parameters at the interaction region to successfully model the detected $\mathrm{x}$-ray beam characteristics. For the case we consider here, the electron beam divergence is comparable to the characteristic $1 / \gamma$ angular width of the $\mathrm{x}$-ray distribution for the case of a single electron, implying that to accurately predict the angular intensity profile, an accurate knowledge of the transverse phase space of the electron beam at the interaction will be required.

In order to determine the electron beam emittance, quadrupole scans were performed upstream of the interaction. In addition, the electron beam energy and energy spread were determined by measuring the dispersionlimited spot size of the electron beam around the dipole bend after the interaction point. The quadrupole scan was performed with a quadrupole magnet placed about $2.0 \mathrm{~m}$ upstream of the interaction. The results of this measurement revealed a somewhat asymmetric beam, with a vertical emittance significantly larger than the horizontal emittance. This ultimately results in a larger vertical divergence of the electron beam at the interaction location. This asymmetry likely originates during the transport and acceleration through the accelerator due to slight misalignments of both the electron beam direction and the linac solenoid axis with respect to the rf axis of the linac sections. Efforts to identify and correct the specific cause of the asymmetry are ongoing.

In principle, the beam parameters determined from the quadrupole scan can be used to calculate the propagation properties through the remainder of the beam line using the known optimized beam line element settings to determine the horizontal and vertical divergences of the electron beam at the interaction. However, errors in the measurement, inaccuracies in the beam line component calibrations, and aberrations in the final focus optics can lead to multiplied inaccuracies in the beam parameters calculated at the interaction point. It is more desirable, therefore, to directly measure the beam parameters at the interaction point. To accommodate this need, the spot size was also obtained by imaging the optical transition radiation produced from an optically finished aluminum cube placed at the interaction point, while the divergence was inferred from measuring the spot size with a yttrium-aluminum garnet (YAG) scintillator around the dipole bend downstream of the interaction point. Because of the relatively large divergence (a few mrad) and the relatively small energy spread (about $0.2 \%$ ), the

(a)
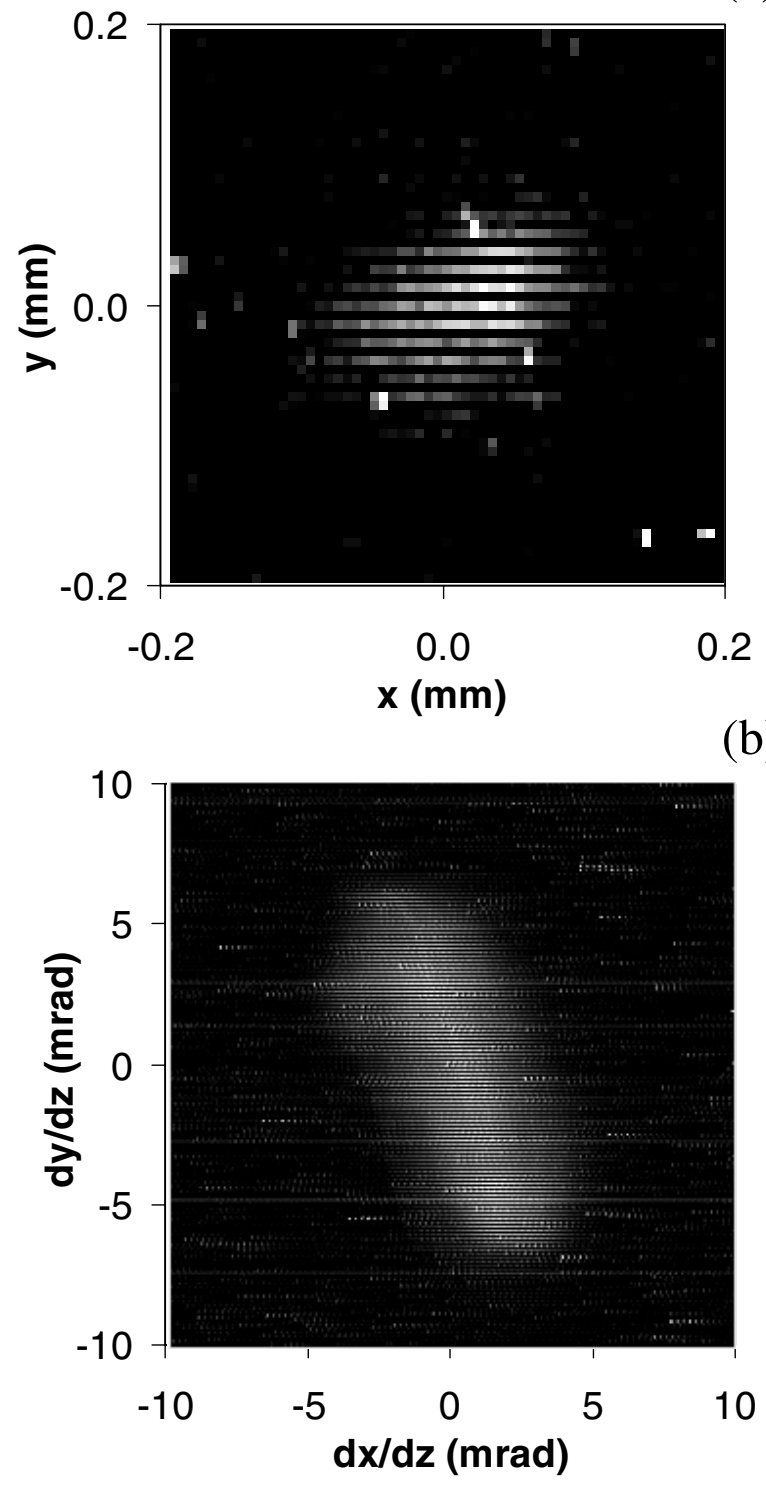

FIG. 3. Optical transition radiation image of the electron beam spot at the interaction (a), and an image from a YAG scintillator taken $0.8 \mathrm{~m}$ downstream of the interaction point used to determine the divergence of the electron beam (b). 
TABLE II. Measured electron beam parameters at the interaction point, including the normalized $\mathrm{rms}$ emittance, the rms spot size, divergence, energy, and energy spread. The electron bunch length was determined from PARMELA simulations of the electron beam production and transport.

\begin{tabular}{ccc}
\hline \hline & $X$ & $y$ \\
\hline$\varepsilon_{n}$ & $4.2 \mathrm{~mm} \mathrm{mrad}$ & $11.2 \mathrm{~mm} \mathrm{mrad}$ \\
$\sigma_{x}$ & $59 \mu \mathrm{m}$ & $38 \mu \mathrm{m}$ \\
$\sigma_{x}^{\prime}$ & $1.8 \mathrm{mrad}$ & $3.1 \mathrm{mrad}$ \\
$E$ & $56.7 \mathrm{MeV}$ & \\
$\sigma_{\gamma}$ & $0.2 \%$ & \\
$\sigma_{t}$ (simulation) & $3 \mathrm{ps}$ & \\
\hline \hline
\end{tabular}

dispersion around the bend can be neglected in determining the divergence from this method. The results of these measurements for the case under consideration is shown in Fig. 3, while a summary of the electron beam parameters is shown in Table II. It should be noted that in Table II the emittance values were determined from the quadrupole scan upstream of the interaction, while the rms spot size and divergence at the interaction were measured independently. While these three values are consistent to within roughly a factor of 2 , in both cases the measured emittance is lower than the emittance suggested by the spot size and divergence measurements. This suggests the possibility of emittance growth during the beam transport and final focus, possibly caused by spherical or chromatic aberrations. The bunch length of the electron beam is roughly $3 \mathrm{ps} \mathrm{rms}$ as determined by
PARMELA simulations of the electron beam production and acceleration, which is consistent with measurements performed with an Imacon 500 Series streak camera of the optical transition radiation produced by the electron bunch.

This second method for determining the electron beam divergence has the additional advantage that rotation of the elliptical electron beam focus about the orthogonal horizontal and vertical axes associated with the quadrupole focus dimensions can be resolved, yielding a more accurate model of the true electron beam phase space. It is seen from the measurement shown in Fig. 3 that the focus is elliptical in nature, which is consistent with the asymmetric emittances measured with the quadrupole scan. However, the ellipse is rotated about $20^{\circ}$ from the horizontal and vertical orthogonal axes, which would not have been apparent from the quadrupole scan results alone. In addition, with the direct measurement of the electron beam parameters, no assumption about the distribution functions describing the electron beam is necessary, allowing the inclusion of effects resulting from non-Gaussian distributions into the simulation of the $\mathrm{x}$-ray beam production.

To input the true electron beam parameters into the 3D time and frequency-domain code, a distribution of macroparticles is reconstructed from the electron beam measurements. If Gaussian distributions can be assumed, it is sufficient for the macroparticle distribution to be derived from a $6 \mathrm{D}$ Gaussian distribution representing the measured rms beam properties shown in Table II, such that

$$
\begin{aligned}
f_{e}(t=0) & =\frac{1}{(2 \pi)^{3} \sigma_{x} \sigma_{y} \sigma_{t} \sigma_{x}^{\prime} \sigma_{y}^{\prime} \sigma_{\gamma}} \exp \left(\frac{-x^{2}}{2 \sigma_{x}^{2}}\right) \exp \left(\frac{-y^{2}}{2 \sigma_{y}^{2}}\right) \exp \left(\frac{-z^{2}}{2 c^{2} \sigma_{t}^{2}}\right) \exp \left(\frac{-x^{\prime 2}}{2 \sigma_{x}^{\prime 2}}\right) \exp \left(\frac{-y^{\prime 2}}{2 \sigma_{x}^{\prime 2}}\right) \exp \left[\frac{-\left(\gamma-\gamma_{0}\right)^{2}}{2 \sigma_{\gamma}^{2}}\right] \\
& =f_{x}(x) f_{y}(y) f_{z}(z) f_{x^{\prime}}\left(x^{\prime}\right) f_{y^{\prime}}\left(y^{\prime}\right) f_{\gamma}(\gamma),
\end{aligned}
$$

where the rms parameters are listed in Table II, and it is assumed that the electron beam is at a waist at $t=0$, with the propagation of each particle in time assumed to be ballistic, and each particle's velocity determined from $x^{\prime}$, $y^{\prime}$, and $\gamma$. This assumption will be valid provided the plasma oscillation period $\left(1 / \omega_{p}\right)$ of the electron beam is much longer than its transit time through the laser pulse, which implies that space-charge effects can be neglected. It is also assumed that there is no correlation between the different components of the distribution, which will automatically be true for $x^{\prime}, y^{\prime}, x$, and $y$ provided the beam is at a waist. On the other hand, correlations between time and energy may very well be present in an actual electron beam, though for the timeintegrated measurements and simulations presented in this paper, these will not be relevant. In general, however, the 3D time and frequency-domain code is well suited to studying the effects of such correlations provided a suitable macroparticle distribution, whether reconstructed from measurements or taken from particle dynamics simulations, is utilized.

In Eq. (11), the $x^{\prime}, y^{\prime}, x$, and $y$ axes have been chosen to line up with the minor and major axes, respectively, of the elliptical distribution. This can be achieved for arbitrary orientations by a simple rotation of coordinates. To model non-Gaussian distributions, a superposition of Gaussian distributions can be implemented, such that for a given coordinate, the distribution can be expressed as

$$
f_{y^{\prime}}\left(y^{\prime}\right)=\sum_{i} \frac{f_{i}}{\sqrt{(2 \pi)} \sigma_{y i}^{\prime}} \exp \left[\frac{-\left(y^{\prime}-y_{0 i}^{\prime}\right)^{2}}{2 \sigma_{y i}^{\prime 2}}\right],
$$

where

$$
\sum_{i} f_{i}=1
$$

In particular, the measured vertical ( $y$ ) divergence of the 
electron beam (Fig. 3) was found to be poorly approximated by a Gaussian distribution. However, a superposition of three Gaussians with appropriate relative amplitudes, angular widths, and average value offsets very closely approximates the measured divergence. This is seen in Fig. 4, which shows very close agreement between the measured distribution function of $d y / d z$ and the analytic expression that was used to generate the macroparticle distribution.

\section{B. Comparison of simulated to measured $x$-ray intensity profile}

The reconstructed electron beam model can now be inserted into the 3D time and frequency-domain code to calculate the expected $x$-ray intensity profile produced in

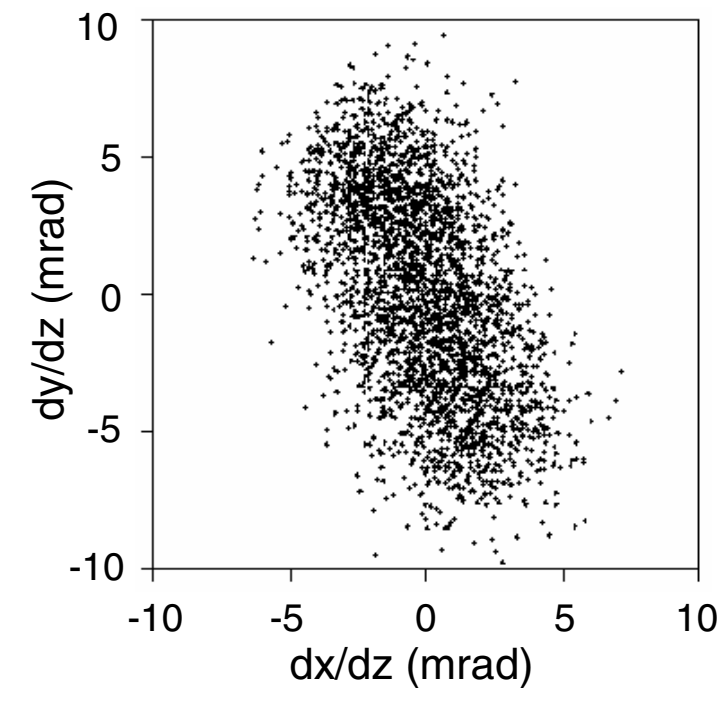

(a)

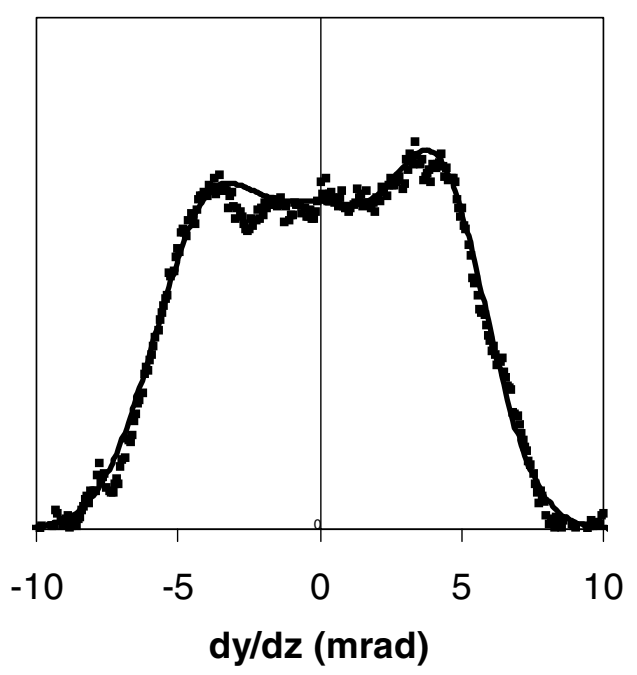

(b)

FIG. 4. Reconstructed macroparticle representation of the electron beam divergence (a), and (b) comparison of the measured $x$ integrated electron beam divergence in $y$ (squares) to the distribution function used to construct the macroparticle distribution. the experiment. To accurately predict the angular size (divergence) and shape of the x-ray beam, the transmission and detection efficiency of the $\mathrm{x}$ rays produced at the interaction will have to be considered. Both the transmission of the $x$ rays though the laser turning optic (Fig. 1) and the response of the CsI scintillator to the incident $\mathrm{x}$ rays have a strong spectral dependence over the spectral range of the source, resulting in a strong dependence of the detected intensity profile on the spectral content of the $\mathrm{x}$-ray pulse. Figure 5 shows both the transmission vs wavelength through the BK7 flat, where the increased width of the flat due to the $40^{\circ}$ incident angle has been taken into account, as well as the interaction probability vs wavelength for the $145 \mu \mathrm{m}$ thick CsI scintillator. Both of these curves must be used in determining the theoretically expected $\mathrm{x}$-ray profile by applying the appropriate
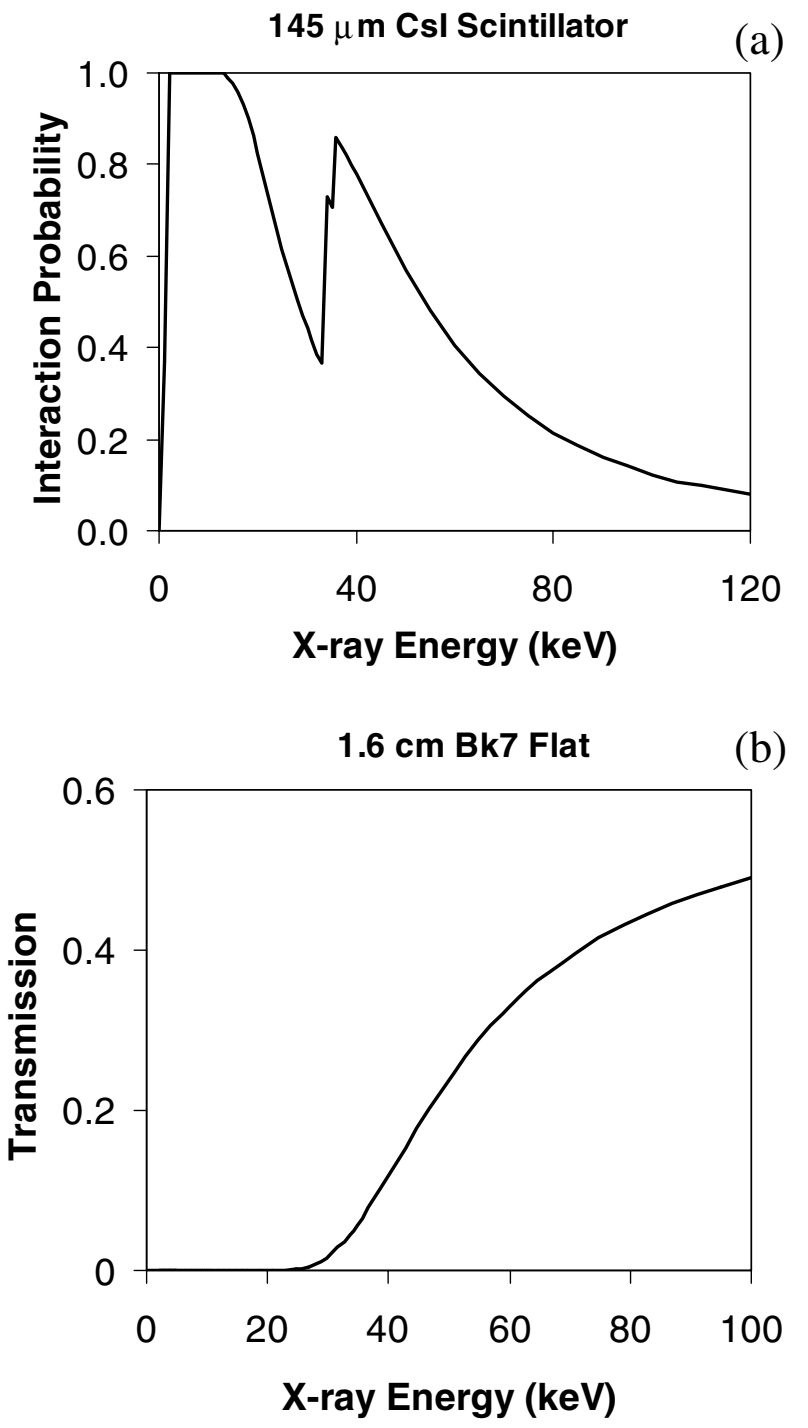

FIG. 5. Interaction probability vs x-ray energy for the CCD scintillator (a), and the transmission probability through the laser turning optic (b). 
attenuation factor for each wavelength being considered. With this modification, Eq. (9) becomes

$$
\frac{d N_{D}\left(\theta, \phi, \omega_{s}, t\right)}{d \Omega d \omega_{s} d t}=P_{t}\left(\omega_{s}\right) P_{d}\left(\omega_{s}\right) \sum_{e} \frac{q_{e}}{e} \frac{d N_{e}\left(\theta_{x e}, \theta_{y e} \theta, \phi, \omega_{s}, t\right)}{d \Omega d \omega_{s} d t}=P_{t}\left(\omega_{s}\right) P_{d}\left(\omega_{s}\right) \frac{d N_{T}\left(\theta, \phi, \omega_{s}, t\right)}{d \Omega d \omega_{s} d t},
$$

where $P_{t}\left(\omega_{s}\right)$ is the probability that a photon of frequency $\omega_{s}$ will be transmitted to the detector, $P_{d}\left(\omega_{s}\right)$ is the probability that the photon is detected, and $N_{D}$ denotes the total number of detected photons.

The theoretical intensity profile can then be obtained by integrating Eq. (14) over $t$ and $\omega_{s}$ for each macroparticle and summing over all the macroparticles in the electron bunch distribution. For the case under consideration, the angular FWHM of the electron distribution (approximately equal to $2.35 \sigma_{x}^{\prime}$ ) is comparable to the FWHM of the intensity distribution for a single electron, approximately equal to $1 / \gamma$. Thus, it is expected that the electron bunch will have a significant effect on the $\mathrm{x}$-ray intensity profile. This is illustrated in Fig. 6, which shows the calculated $\mathrm{x}$-ray profile for the measured electron bunch colliding with a laser pulse polarized in both the horizontal (corresponding to the experimental case) and the vertical planes. In the case of the vertically polarized laser pulse, the elongation of the x-ray profile in the horizontal dimension is largely counteracted by the larger focus angle of the electron bunch in the vertical dimension, resulting in a fairly symmetric looking profile. On the other hand, with the laser pulse polarized in the horizontal dimension, the elongation effects of the polarization and the electron beam focus add, resulting in a predicted $\mathrm{x}$-ray profile much like the measured profile (shown in Fig. 2).

A direct comparison of the measured and theoretical intensity profiles is shown in Fig. 7, displaying excellent agreement between the measured and calculated profiles. These were obtained by taking a line out along the major and minor axes of the measured (Fig. 2) and simulated [Fig. 6(a)] intensity profiles. The slight differences between the theoretical and the measured widths can possibly be explained by errors in the electron beam emittance and energy determination, affecting both the width and the spectrum of the x-ray pulse. In addition, if the electron beam focus is sufficiently far from the interaction, from either unoptimized quadrupole settings and/ or timing errors between the electron and laser pulses, only the smaller convergence angles will be sampled by the laser pulse, resulting in less broadening of the $x$-ray profile due to the electron beam divergence.

\section{C. $K$-edge absorption observation}

To further test the validity of the $3 \mathrm{D}$ time and frequency-domain code, the effects of $K_{\alpha}$ absorption near the peak scattered $x$-ray wavelength on the measured intensity profile were observed and compared to that predicted by simulation. This was performed by placing a $125 \mu \mathrm{m}$ Ta foil in the path of the $\mathrm{x}$-ray beam. The transmission characteristics for the Ta foil are shown in Fig. 8. If the electron beam energy is tuned such that the on-axis scattered photon energy is near the $K_{\alpha}$ absorption edge ( $K$ edge), the observed intensity profile will be very sensitive to the produced spectrum.

We consider two cases of $K$-edge absorption. In one, the electron beam energy was tuned to $55 \mathrm{MeV}$, resulting in a peak on-axis photon energy of $73.1 \mathrm{keV}$, and in the

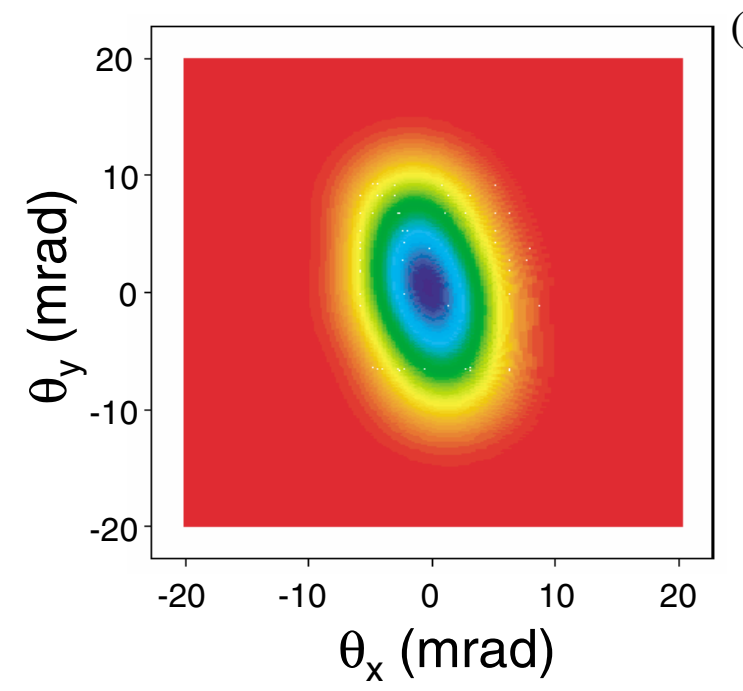

(a)

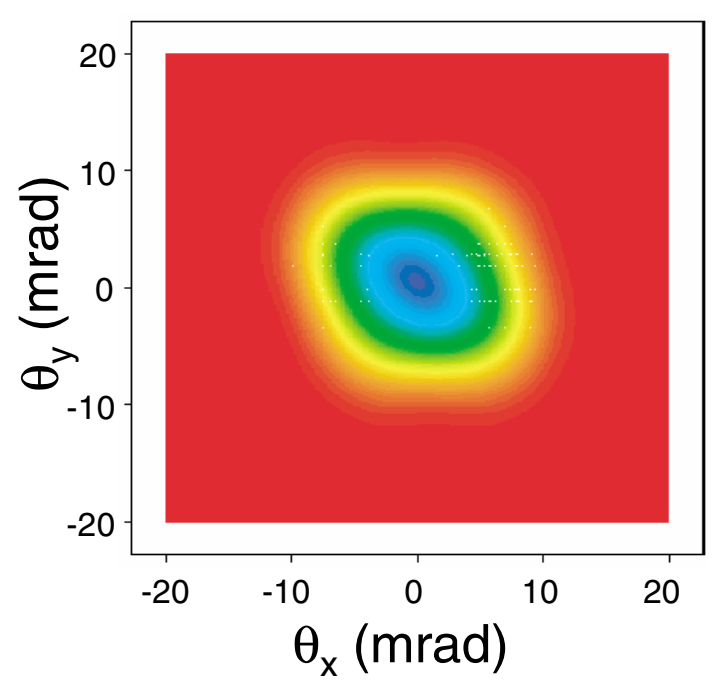

(b)

FIG. 6. (Color) Theoretical intensity profiles determined from the measured laser and electron beam parameters for the case of an $x$ polarized incident laser pulse (a), which corresponds to the measured case (Fig. 2), and a $y$ polarized laser pulse (b). 
(a)

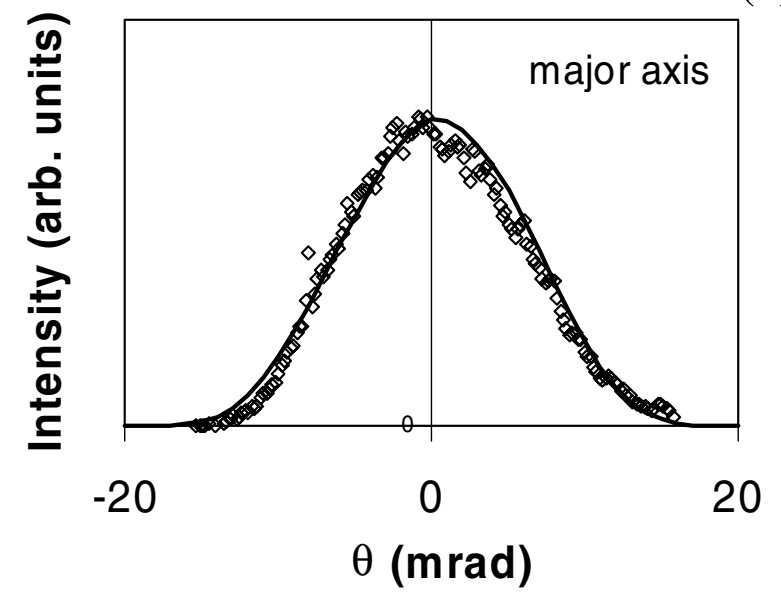

(b)

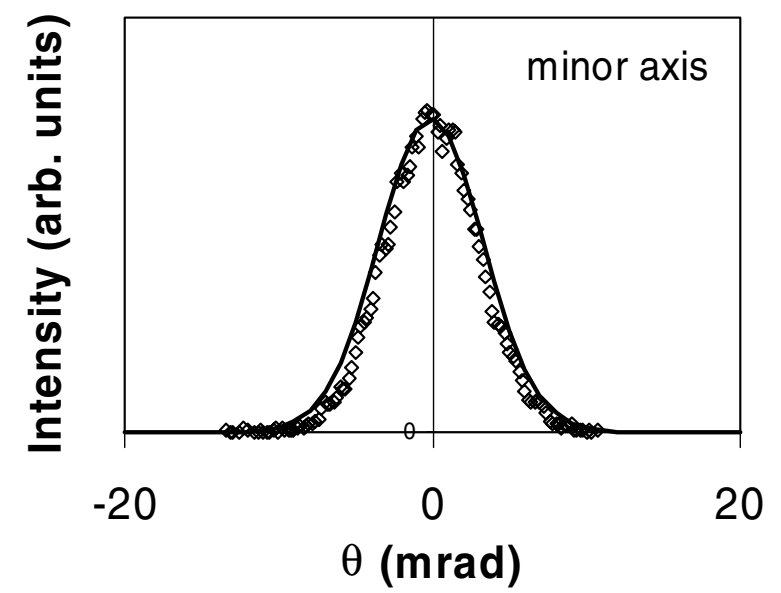

FIG. 7. Measured (dots) and simulated (line) intensity profile along the major (a) and minor (b) axes of the Thomson $\mathrm{x}$-ray beam.

other, the electron energy was $57 \mathrm{MeV}$, resulting in a peak on-axis photon energy of $78 \mathrm{keV}$. To resolve the $K$-edge absorption in the $\mathrm{x}$-ray intensity profile, the electron beam transport and final focus optics were tuned to a smaller electron beam convergence angle at the interaction, which results in a larger electron beam spot size, but increases the correlation between the x-ray wavelength and the observation angle. For both electron beam energies, the rms divergence of the electron beam in the horizontal and vertical dimensions were $0.9 \mathrm{mrad}$ and $1.3 \mathrm{mrad}$, respectively.

In the first case, the peak photon energy on axis is only slightly above the $K$-edge energy of $68 \mathrm{keV}$, resulting in a large degree of attenuation in the center portion of the detected intensity profile. A measurement of a 100 shot integrated intensity profile for this case is shown in Fig. 9(a). In the second case, the on-axis photon energy

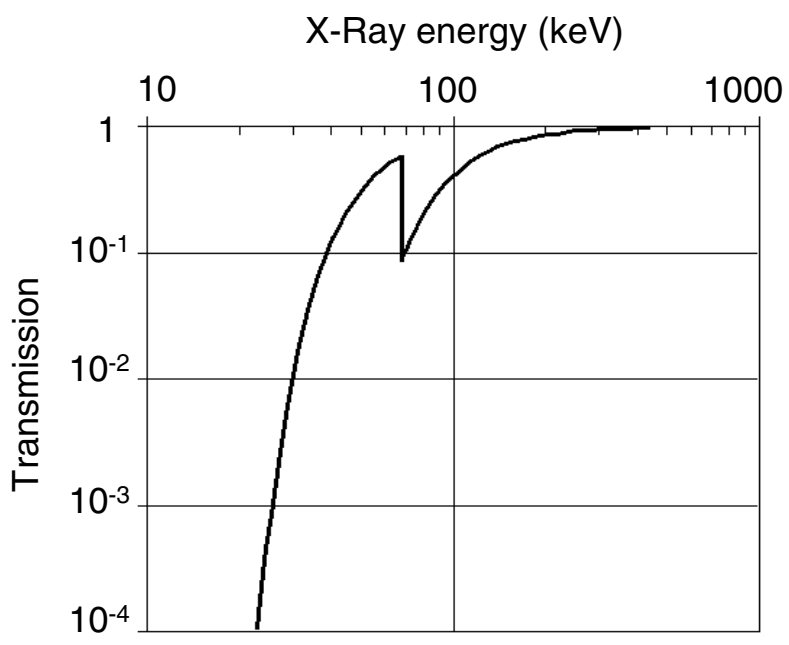

FIG. 8. Transmission probability through a $0.125 \mathrm{~mm}$ thick Ta foil.

is sufficiently far above the $K$-edge such that most photons are transmitted through the foil, while photons slightly off axis are attenuated. This results in a detected intensity profile roughly described by a dark ring surrounding a bright center [Fig. 10(a)]. Simulations of both cases are shown in Figs. 9(b) and 10(b). It was found that excellent agreement with the measured profile was obtained provided the electron beam energy in the simulation was about $1 \mathrm{MeV}$ higher than the measured electron beam energy. This slight discrepancy is most likely explained by systematic errors in the electron beam energy measurement due to alignment and field calibration errors of the dipole magnet. In this respect, the x-ray measurements in conjunction with the detailed information provided by the 3D time and frequency-domain code can provide an important check of the electron beam diagnostics. The use of Thomson scattered $\mathrm{x}$ rays as a tool for diagnosing electron beam properties has previously been implemented by Leemans et al. [16].

\section{X-RAY BEAM FLUX AND BRIGHTNESS DETERMINATION}

The good agreement between the theoretical and the measured intensity profiles and $K$-edge transmission characteristics provides good evidence that the spatially correlated spectral content of the Thomson scattered $\mathrm{x}$ rays calculated by the $3 \mathrm{D}$ time and frequency-domain code is accurate. Hence, we can confidently utilize this information in extrapolating from the integrated intensity of the CCD image to infer the number of photons produced in the interaction, as well as $\mathrm{x}$-ray flux and spectral brightness of the source.

Because of the spectrally dependent transmission of the x-ray beam to the detector, $P_{t}(\omega)$, and the detection sensitivity, $\eta(\omega)$, the energy detected by the CCD will depend greatly on the spectral content of the x-ray beam. 
Theoretically, the total number of counts in the integrated CCD image will be given by

$$
N_{\mathrm{CCD}}=\sum_{e} \int\left[P_{T}\left(\omega_{s}\right) \eta\left(\omega_{\mathrm{s}}\right) \hbar \omega_{s} \frac{q_{e}}{e} \iint \frac{d N_{e}\left(\theta_{x e}, \theta_{y e}, \theta, \phi, \omega_{s}, t\right)}{d \Omega d \omega_{s} d t} d \Omega d t\right] d \omega_{s},
$$

where the detection sensitivity, $\eta(\omega)$, in units of counts/unit photon energy, can be expressed in terms of the sensitivity at the calibrated photon energy $\omega_{c}$ :

$$
\eta(\omega)=\eta\left(\omega_{c}\right) \frac{P_{D}(\omega)}{P_{D}\left(\omega_{c}\right)}
$$

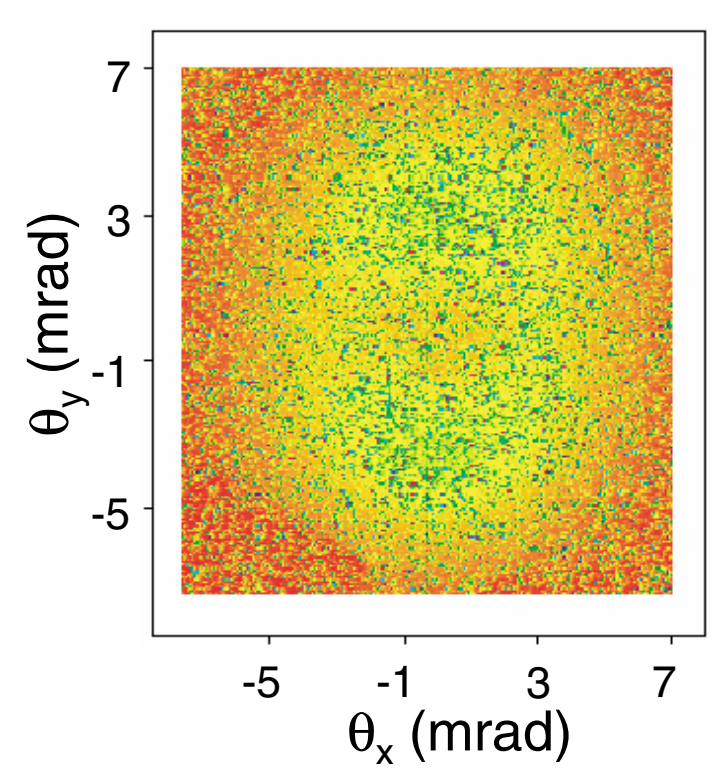

(a)

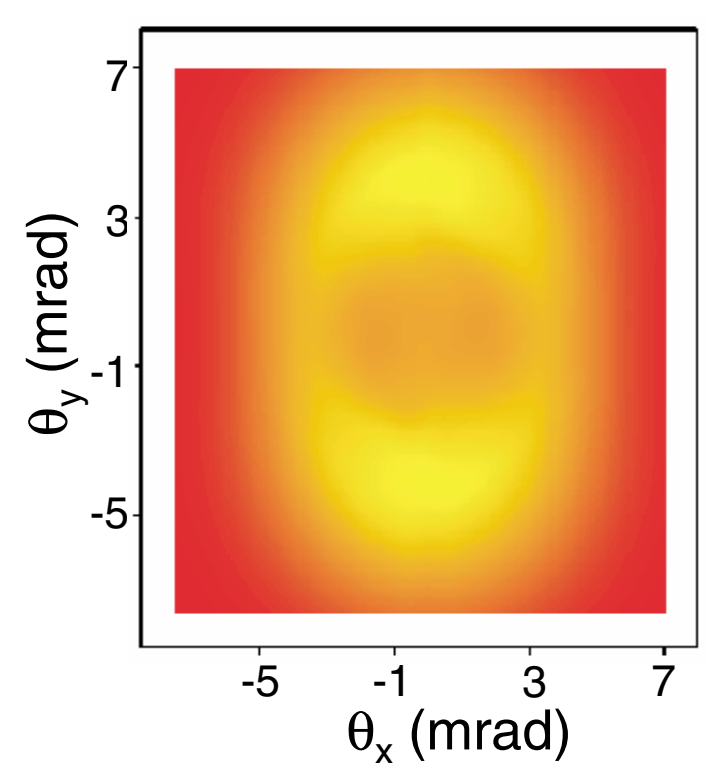

(b)

FIG. 9. (Color) Measured (a) and simulated (b) intensity profiles for the transmission of $\mathrm{x}$ rays through a $0.125 \mathrm{~mm}$ Ta foil. The measured electron beam energy was $55 \mathrm{MeV}$, while the energy used in the simulation was $56 \mathrm{MeV}$.
Equation (15) is simply Eq. (14) integrated over solid angle, time, and photon energy, and multiplied by the constant $\eta\left(\omega_{c}\right) / P_{D}\left(\omega_{c}\right)$. The total number of photons produced at the interaction, $N_{T}$, is given by

(a)

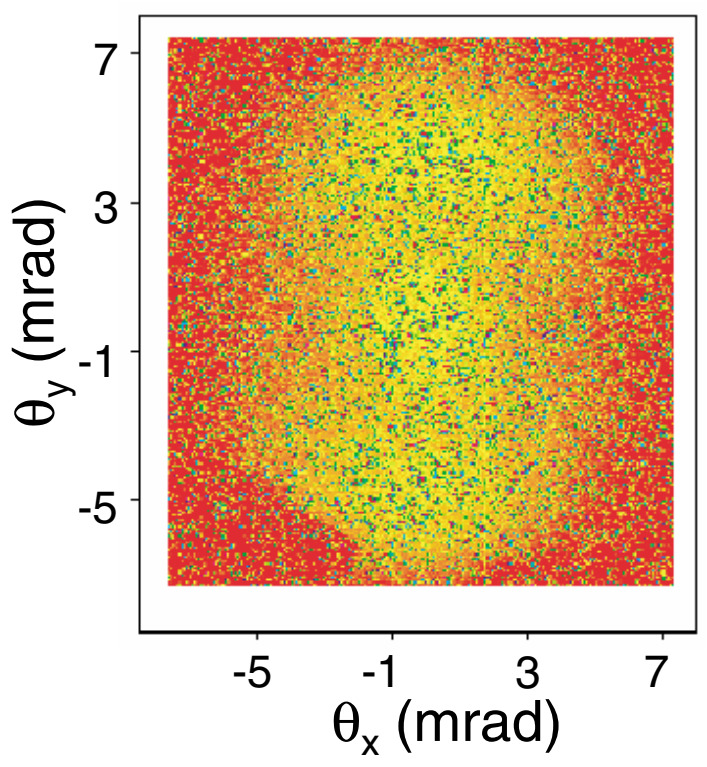

(b)

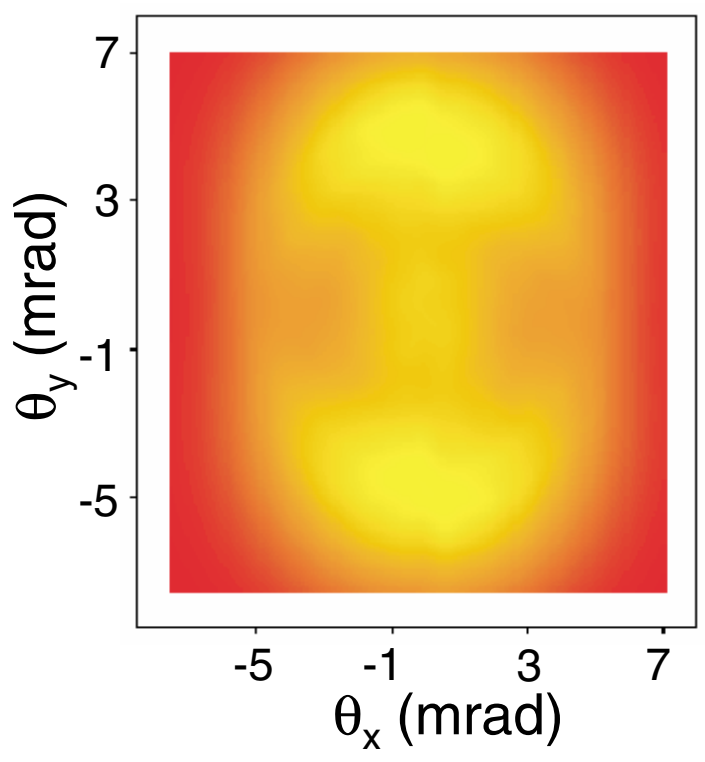

FIG. 10. (Color) Measured (a) and simulated (b) intensity profile for $\mathrm{x}$-rays transmitted through a $0.125 \mathrm{~mm}$ Ta foil. The measured electron beam energy was $57 \mathrm{MeV}$, while the energy used in the simulation was $58 \mathrm{MeV}$. 
TABLE III. Measured x-ray beam characteristics. Source size and divergence parameters are the root mean square intensity values. The source size is inferred from measurements of the laser spot size and electron spot size. The spectral information is inferred from simulations of the Thomson interaction based on measurements of the electron beam characteristics, and the photon count and divergence is inferred from the CCD image of the X-ray beam (Fig. 2).

\begin{tabular}{lc}
\hline \hline \multicolumn{1}{c}{ Parameter } & Value \\
\hline Divergence $(x)$ & $3.1 \mathrm{mrad}$ \\
Divergence $(y)$ & $5.8 \mathrm{mrad}$ \\
Source size & $0.018 \mathrm{~mm}$ \\
Peak on axis photon energy & $75 \mathrm{keV}$ \\
On axis bandwidth (FWHM) & $12.5 \mathrm{keV}$ \\
Average photon energy & $37 \mathrm{keV}$ \\
Single shot photon count & $4.4 \times 10^{6}$ \\
Peak photon flux & $6 \times 10^{18} \mathrm{photons}_{\mathrm{s}}$ \\
Peak spectral brightness & $5 \times 10^{15} \mathrm{photons}_{\left(\mathrm{s} \mathrm{mm}^{2} \mathrm{mrad}^{2} 0.1 \% \text { b.w. }\right)}$ \\
\hline \hline
\end{tabular}

$$
N_{T}=\sum_{e} c\left|\frac{\mathbf{k}}{k_{0}}-\boldsymbol{\beta}_{e}\right| \sigma_{T} \frac{q_{e}}{e} \int n_{\gamma}\left(\mathbf{r}_{e}(t), t\right) d t .
$$

Note that Eq. (17) is a relatively fast calculation to perform, since it depends only on the time integration of the product of the electron and photon flux multiplied by the total Thomson cross section, with no need to integrate over solid angles or scattered frequency. From the computation of the ratio of Eq. (17) to Eq. (15), which will be defined as $\kappa$, the total photon dose in the x-ray pulse at the interaction point can be easily determined from the integrated CCD image such that

$$
N_{T}=\kappa N_{\mathrm{CCD}}
$$

where

$$
\kappa=\frac{\sum_{e} c\left|\frac{\mathbf{k}}{k_{0}}-\boldsymbol{\beta}_{e}\right| \sigma_{T} \frac{q_{e}}{e} \int n_{\gamma}\left(\mathbf{r}_{e}(t), t\right) d t}{\frac{\eta\left(\omega_{c}\right)}{P_{D}\left(\omega_{c}\right)} \iiint \hbar \omega_{s} \frac{d N_{D}\left(\theta, \phi, \omega_{s}, t\right)}{d \Omega d \omega_{s} d t} d \Omega d t d \omega_{s}} .
$$

Likewise, the total energy in the $\mathrm{x}$-ray pulse can be expressed as

$$
U_{T}=\left\langle\hbar \omega_{s}\right\rangle \kappa N_{\mathrm{CCD}}
$$

where

$$
\left\langle\hbar \omega_{s}\right\rangle=\frac{1}{N_{T}} \iiint \hbar \omega_{s} \frac{d N_{D}\left(\theta, \phi, \omega_{s}, t\right)}{d \Omega d \omega_{s} d t} d \Omega d t d \omega_{s}
$$

is the average photon energy in the x-ray pulse. For the case under consideration, these calculations result in $\kappa=$ 0.98 photons/count. For the measured x-ray intensity profile shown in Fig. 2, this results in a measured total photon dose of $N_{T}=4.4 \times 10^{6}$, with an average photon energy given by $\left\langle\hbar \omega_{s}\right\rangle=37.2 \mathrm{keV}$. The properties of the measured $x$-ray beam are summarized in Table III.

Finally, the peak brightness of the measured x-ray pulse can be obtained from the time-dependent spectra determined from the 3D time and frequency-domain code in conjunction with the measured $\mathrm{x}$-ray dose. The time duration of the $\mathrm{x}$-ray pulse closely mimics the duration of the electron bunch due to the head collision geometry, resulting in an $\mathrm{x}$-ray pulse duration of about $3 \mathrm{ps} \mathrm{rms}$, and a peak photon flux of about $6 \times 10^{18}$ photons/s. The source size is the result of the convolution of the electron and laser spot sizes at the interaction and is roughly equal to $18 \mu \mathrm{m}$ rms. The calculated time-dependent spectrum is shown in Fig. 11, where a peak spectral brightness of about $5 \times 10^{15}$ photons $/\left(\mathrm{s} \mathrm{mm}^{2} \mathrm{mrad}^{2} 0.1 \%\right.$ b.w. $)$ is predicted.

Note that while $N_{\mathrm{CCD}}$ is the only directly measured quantity in Eq. (17), both the values for $\kappa$ and $\left\langle\hbar \omega_{s}\right\rangle$ are relatively insensitive to the details of the electron beam and laser beam distributions. This is due to the fact that the quantities are integrated over all wavelengths and observation angles, tending to wash out the effects of the spectral broadening in any given observation

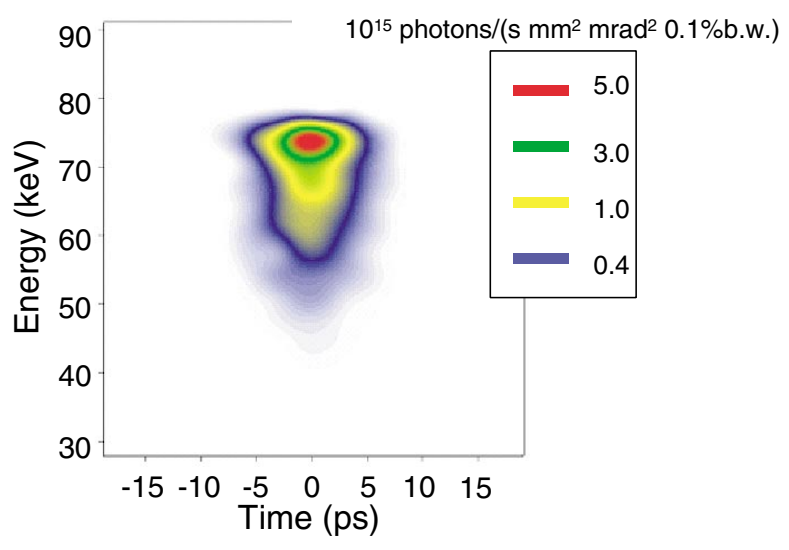

FIG. 11. (Color) On axis time-dependent $\mathrm{x}$-ray spectrum of the experimentally produced $\mathrm{x}$-ray beam as determined by the $3 \mathrm{D}$ time and frequency-domain code. 
direction. To a high degree of accuracy, these quantities can be simply calculated from the single electron scattering cross section and angular spectral dependence described by Eqs. (1) and (2), where $\omega_{0}$ is now the average laser frequency, and $\gamma$ is the average electron beam Lorentz factor. Thus, it can be concluded that in the regime being considered here, $\kappa$ and $\left\langle\hbar \omega_{s}\right\rangle$ are primarily functions of the overall electron beam energy, laser wavelength, and interaction geometry, all of which can be experimentally determined with a high degree of confidence. This, along with the excellent agreement between the simulated and measured x-ray beam characteristics, suggests that the parameters listed in Table III are accurate estimates of the true $\mathrm{x}$-ray beam properties.

\section{CONCLUSIONS}

Measured electron and $\mathrm{x}$-ray beam parameters have been used to benchmark a newly developed threedimensional time and frequency-domain code designed to provide complete 3D time-resolved computational capability for the full determination of the temporal and spatially resolved spectra and intensity distributions produced from a Thomson interaction of arbitrary geometry. This capability is crucial for both the design of successful Thomson x-ray sources, as well as future experiments and applications utilizing such sources. The measured intensity profile of the x-ray beam produced from the collision of a $3 \mathrm{ps}, 55 \mathrm{MeV}$ electron beam with a $50 \mathrm{fs}$, $800 \mathrm{~nm}$ laser pulse was found to agree very well with that predicted from simulations with the new code after the inclusion of the spectrally dependent transmission and the detection efficiency of the $x$ rays. In addition, the spatial structure of the measured $\mathrm{x}$-ray intensity profile induced by $K_{\alpha}$ absorption through a Ta foil agreed very well with simulations. The input parameters of the simulation were determined by careful measurements of the electron beam energy, energy spread, spot size, and divergence at the interaction point. Thus, it can be inferred that time-integrated spatially correlated spectra predicted by the code is accurate. The simulated $x$-ray spectrum and overall detection efficiency predicted by the code was utilized to estimate the total $\mathrm{x}$-ray dose in the measured beam to be about $4 \times 10^{6}$ photons. The peak flux and peak spectral brightness of the measured beam were determined to be $6 \times 10^{18}$ photons/s and $5 \times 10^{15}$ photons $/\left(\mathrm{s} \mathrm{mm}^{2} \mathrm{mrad}^{2} 0.1 \%\right.$ b.w. $)$ respectively. Finally, we note that a full description of the theory behind the 3D time and frequency-domain code, as well as detailed calculations of femtosecond $\mathrm{x}$-ray pulse production through Thomson scattering, is presented in a companion paper.

\section{ACKNOWLEDGMENTS}

This work was performed under the auspices of the U.S. Department of Energy by the University of California, Lawrence Livermore National Laboratory under Contract No. W-7405-Eng-48. UCRL-JP200521.

[1] A. H. Chin et al., Phys. Rev. Lett. 83, 336 (1999).

[2] A. M. Lindenberg et al., Phys. Rev. Lett. 84, 111 (2000).

[3] J. N. Galayda, in Proceedings of the 8th International Conference on X-Ray Lasers, Aspen, CO, 2002 (AIP, Melville, NY, 2002), p. 365.

[4] R.W. Schoenlein et al., Science 274, 236 (1996).

[5] W. P. Leemans et al., Phys. Rev. Lett. 77, 4182 (1996).

[6] S. Kashiwagi et al., Nucl. Instrum. Methods Phys. Res., Sect. A 455, 36 (2000).

[7] I.V. Pogorelsky et al., AIP Conf. Proc. 569, 571 (2001).

[8] A. E. Vlieks, G. Caryotakis, W. R. Fowkes, E. N. Jongewaard, E. C. Landahl, R. Loewen, and N.C. Luhmann, AIP Conf. Proc. 625, 107 (2002).

[9] R. P. Fischer, A. Ting, C. I. Moore, P. Sprangle, M. Baine, R. Elton, and S. Ride, in Proceedings of the 2001 Particle Accelerator Conference, Chicago, IL (IEEE, Piscataway, NJ, 2001), p. 2644.

[10] A. Fukasawa et al., Int. J. Appl. Electromag. Mech. 14, 221 (2001).

[11] D. J. Gibson et al., Phys. Plasmas 11, 2857 (2004).

[12] T. Guo, C. H. Spielmann, B. C. Walker, and C. P. J. Barty, Rev. Sci. Instrum. 72, 41 (2001).

[13] J. Arthur, G. Materlik, R. Tatchyn, and H. Winick, Rev. Sci. Instrum. 66, 1987 (1995).

[14] F.V. Hartemann et al., Phys. Rev. E 64, 016501 (2001).

[15] G. A. Krafft, in Proceedings of the 1997 Particle Accelerator Conference (IEEE, Piscataway, NJ, 1998), Vol. 1, p. 739.

[16] W. P. Leemans et al., IEEE J. Quantum Electron. 33, 1925 (1997).

[17] S. K. Ride, E. Esarey, and M. Baine, Phys. Rev. E 52, 5425 (1995).

[18] F.V. Hartemann and A. K. Kerman, Phys. Rev. Lett. 76, 624 (1996).

[19] E. Esarey, S. K. Ride, and P. Sprangle, Phys. Rev. E 48, 3003 (1993).

[20] C. Bula et al., Phys. Rev. Lett. 76, 3116 (1996).

[21] K. Moffat, Faraday Discuss. Chem. Soc. 122, 65 (2002).

[22] C. B. Schroeder et al., Nucl. Instrum. Methods Phys. Res., Sect. A 483, 89 (2002).

[23] H. N. Chapman and K. A. Nugent, Opt. Commun. 205, 351 (2002).

[24] C. Pellegrini, Nucl. Instrum. Methods Phys. Res., Sect. A 445, 124 (2000).

[25] W. J. Brown and F.V. Hartemann (to be published).

[26] J. B. Rosenzweig, Fundamentals of Beam Physics (Oxford University Press, New York, 2003), p. 215.

[27] F.V. Hartemann, High-Field Electrodynamics (CRC Press, Boca Raton, FL, 2002), p. 453.

[28] M. D. Perry and G. Mourou, Science 264, 917 (1994). 